\title{
Nonlinear Inertia Classification Model and Application
}

\author{
Mei Wang, ${ }^{1}$ Pai Wang, ${ }^{1}$ Jzau-Sheng Lin, ${ }^{2}$ Xiaowei Li, ${ }^{1}$ and Xuebin Qin ${ }^{1}$ \\ ${ }^{1}$ College of Electric and Control Engineering, Xi'an University of Science and Technology, Xi'an 710054, China \\ ${ }^{2}$ Department of Computer Science and Information Engineering, National Chin-Yi University of Technology, Taichung 41170, Taiwan
}

Correspondence should be addressed to Jzau-Sheng Lin; jslin@ncut.edu.tw

Received 26 February 2014; Accepted 24 April 2014; Published 22 May 2014

Academic Editor: Her-Terng Yau

Copyright (C) 2014 Mei Wang et al. This is an open access article distributed under the Creative Commons Attribution License, which permits unrestricted use, distribution, and reproduction in any medium, provided the original work is properly cited.

\begin{abstract}
Classification model of support vector machine (SVM) overcomes the problem of a big number of samples. But the kernel parameter and the punishment factor have great influence on the quality of SVM model. Particle swarm optimization (PSO) is an evolutionary search algorithm based on the swarm intelligence, which is suitable for parameter optimization. Accordingly, a nonlinear inertia convergence classification model (NICCM) is proposed after the nonlinear inertia convergence (NICPSO) is developed in this paper. The velocity of NICPSO is firstly defined as the weighted velocity of the inertia PSO, and the inertia factor is selected to be a nonlinear function. NICPSO is used to optimize the kernel parameter and a punishment factor of SVM. Then, NICCM classifier is trained by using the optical punishment factor and the optical kernel parameter that comes from the optimal particle. Finally, NICCM is applied to the classification of the normal state and fault states of online power cable. It is experimentally proved that the iteration number for the proposed NICPSO to reach the optimal position decreases from 15 to 5 compared with PSO; the training duration is decreased by $0.0052 \mathrm{~s}$ and the recognition precision is increased by $4.12 \%$ compared with SVM.
\end{abstract}

\section{Introduction}

Power cables play an extremely important role in industrial production and modern life. At present, it is difficult for people to accept a bank system chaos or a wrong airport management system because of the power cable faults. In order to decrease and avoid the economic loss, the correct state classification of the online power cable is very necessary.

Nowadays, the commonly used fault diagnosis methods of power cable are electrical bridge method and electrical impulse method. Both of these methods are offline methods [1]. Obviously, these offline methods cannot satisfy the requirement.

In theory, the entropy of the zero-sequence components [2] of 3-phase voltages and 3-phase currents of the online power cable was used to extract the fault feature. In addition, wavelet transform is useful for the feature extraction of the early fault of the online cable, and then the voltages and currents are detected to get the subtle singular points by utilizing wavelet transform [3]. Furthermore, the artificial neural network [4] was also used to build the state classification model of the power cable because it can realize any nonlinear mapping. But artificial neural network needs a large number of samples and the training process may go to the local minimum point. The classification model of support vector machine (SVM) overcomes the disadvantage of big samples by obeying the rule of the minimum structural risk [5]. But the kernel parameter and the punishment factor have great influence on the quality of SVM model.

Particle swarm optimization (PSO) is an evolutionary search algorithm [6] based on the swarm intelligence, which is suitable for parameter optimization. Therefore, the combination of PSO and SVM can find the optimal kernel parameter and the punishment factor of SVM and obtain a high quality of SVM classification model.

This paper is organized as follows. After the introduction in Section 1, SVM and PSO related to this study are given in Section 2. It includes the conventional SVM and the traditional PSO as well as the specific PSO with a convergence factor and an inertia factor. Then, the nonlinear inertia convergence PSO (NICPSO) and the nonlinear inertia convergence classification model (NICCM) are proposed in Section 3. The experiments are implemented using NICPSO and NICCM to classify the normal state and several fault states of online power cable in Section 4. Finally, the conclusion is obtained in Section 5. 


\section{SVM and PSO}

The basic idea of NICCM is that SVM is taken as the classification model and PSO is taken to optimize the important parameters of the punishment factor and the kernel parameter of SVM. The principles of SVM and PSO are reviewed below.

2.1. SVM. The principle of SVM is to find an optimal classification hyperplane which separates as much as possible patterns of two classes to the correct classes. Meanwhile, the hyperplane ensures the maximum distance between the two classes of separable samples [7]. If $d_{+}$is the minimum distance from the classification hyperplane to the positive sample set, and $d_{-}$is the minimum distance from the classification hyperplane to the negative sample set, then the margin of the classification hyperplane is " $d_{+}+d_{-}$". The linear SVM is to find the separation hyperplane with maximum margin. Namely, all the training samples should satisfy the following constraints of $\left|d_{+}\right|=\left|d_{-}\right|=1$ :

$$
\begin{aligned}
& x_{i}^{T} w+b \geq+1, \quad y_{i}=+1, \quad i=1,2, \ldots, N \\
& x_{i}^{T} w+b \leq-1, \quad y_{i}=-1, \quad i=1,2, \ldots, N
\end{aligned}
$$

or the equivalent constraint

$$
y_{i}\left(x_{i}^{T} w+b\right)-1 \geq 0, \quad \forall i
$$

where $x_{i}$ is the $i$ th training sample and $y_{i}$ is the class label of the training sample $x_{i} ; w$ and $b$ are the parameters of the classification hyperplane; $T$ represents the transposition of a vector; $N$ is the number of samples.

If a training sample satisfies (2), then it is a support vector. The change of a support vector impacts the change of the margin and the solution of problem.

Linear support vector machine is a maximization problem in the view of (2). Equivalently, it is a minimum problem of $\|w\|^{2}$ in the condition of (2); namely, it is an optimization problem with the constraint

$$
\begin{aligned}
& J=\min \left\{\frac{1}{\left[2 *\|w\|^{2}\right]}\right\} \\
& \text { s.t. } y_{i}\left(x_{i}^{T} w+b\right)-1 \geq 0, \quad \forall i
\end{aligned}
$$

where $x_{i}$ is the $i$ th training sample and $y_{i}$ is the class label of the training sample $x_{i}$ and $w$ and $b$ are the parameters of the classification hyperplane. This is a typical convex quadratic programming problem.

For the linear separable classification problem of twoclass, the minimum structural risk problem can be described by the conditional quadratic optimization problem. SVM perfectly solves the two-class classification problem by finding the solution of a convex quadratic optimization problem [8].

For the indivisible linear problem of two-class, SVM reaches the optimal result by constructing a classification hyperplane with a soft margin. According to Mercer kernel expansion theorem, a sample space can be mapped to a higher dimensional feature space in which the linear learning machine can be used to solve the nonlinear classification problem. The solution of inseparable linear two-class problem makes SVM become one of the formal two-class classifiers with two values [9].

Given a sample set $T, T=\left\{x_{i}, y_{i} \mid i=1,2, \ldots, N\right\} . x_{i}$ is an input vector; $y_{i} \in\{+1,-1\}$ denotes the corresponding desired output vector; $N$ is the sample number. The Lagrange multiplier $\alpha$ is introduced to construct the optimal classification function $f(x)$ of separable linear samples; then

$$
f(x)=\operatorname{sgn}\left(w^{*} \cdot x+b^{*}\right)=\operatorname{sgn}\left\{\sum_{i=1}^{l} y_{i} \alpha_{i}^{*}\left(x \cdot x_{i}\right)+b^{*}\right\},
$$

where sgn is the sign function. The class of pattern $x$ is determined by the sign of the brackets.

For the case of imperfect separable linear samples, the loss introduced by classification error should be considered. The relax factor $\varepsilon_{i} \geq 0, i=1, \ldots, N$, is used to (2). There is

$$
y_{i}\left(x_{i}^{T} w+b\right)-1+\varepsilon_{i} \geq 0, \quad \forall i .
$$

Then the classification hyperplane with a soft margin is determined by the optimization problem

$$
\begin{aligned}
& J=\min \left\{\frac{1}{\left[2 *\|w\|^{2}\right]}+C \sum_{i=1}^{l} \varepsilon_{i}\right\} \\
& \text { s.t. } y_{i}\left(x_{i}^{T} w+b\right)-1+\varepsilon_{i} \geq 0, \quad i=1, \ldots, N,
\end{aligned}
$$

where $w$ and $b$ are, respectively, the weights and constant of classification hyperplane; $C$ is the punishment factor of wrong classification which adjusts the balance between the confidence range and the experienced error. The bigger $C$ implies the smaller experienced error, and the smaller $C$ means the bigger classification margin. In addition, $C$ is a positive constant, and the bigger $C$ means the more serious punishment.

To solve (6), Lagrange multiplier $\alpha=\left(\alpha_{1}, \alpha_{2}, \ldots, \alpha_{l}\right)^{T}$ and Lagrange function $L$ are used to convert the problem (6) to a quadratic programming problem

$$
\begin{aligned}
& L=\sum_{i=1}^{N} \alpha_{i}-\frac{1}{2} \sum_{i=1}^{N} \alpha_{i} \alpha_{j} y_{i} y_{j} x_{i}^{T} x_{j}, \quad 0 \leq \alpha_{i} \leq C, i=1, \cdots, N \\
& \text { s.t. } \sum_{i=1}^{N} \alpha_{i} y_{i}=0 .
\end{aligned}
$$

Most of the practical problems are nonlinear. Then kernel function $K\left(x_{i}, x_{j}\right)=\left\langle\Phi\left(x_{i}\right), \Phi\left(x_{j}\right)\right\rangle$ is introduced to convert an inseparable linear problem in a lower dimensional space to a separable linear problem in a higher dimensional space. In this case, the quadratic programming problem is

$$
L=\sum_{i=1}^{N} \alpha_{i}-\frac{1}{2} \sum_{i=1}^{N} \sum_{j=1}^{N} \alpha_{i} \alpha_{j} y_{i} y_{j} K\left(x_{i}, x_{j}\right) \text {. }
$$


So the nonlinear SVM classifier $f(x)$ is

$$
f(x)=\operatorname{sgn}\left\{\sum_{i=1}^{N} y_{i} \alpha_{i} K\left(x, x_{i}\right)+b\right\},
$$

where sgn is the sign function and $K\left(x, x_{i}\right)$ is a kernel function. The commonly used kernel functions are linear function, polynomial function, Sigmoid function, and Gauss redial basis kernel function which is used in this paper. Gauss redial basis kernel function is given as follows:

$$
K\left(x_{i}, x_{j}\right)=e^{-\left\|x_{i}-x_{j}\right\|^{2} /\left(2 \sigma^{2}\right)},
$$

where $x_{i}$ and $x_{j}$ are, respectively, the $i$ th and $j$ th sample; $\sigma$ is the width of redial basis function.

The punishment factor $C$ controls the punishment degree by which the sample with over error is penalized [10]. Besides, the generalization ability of SVM is determined by the kernel parameter $\sigma$ [11]. That is to say, these two parameters have great influence on the quality of SVM classification model.

2.2. PSO. The basic idea of PSO is to take every particle with a random initialization as a possible solution of optimization problem, and the quality of the particle is determined by a predefined fitness function. Each particle moves in the possible solution space, and the direction and the distance are determined by a velocity variable. Particles generally follow the current optimal particle, and the optimal solution will be obtained finally through each generation of searching [12]. If $p_{i}=\left(p_{i 1}, p_{i 2}, \ldots, p_{i D}\right)$ is the most optimal position which has been experienced by the individual particle, and $p_{g}=\left(p_{g 1}, p_{g 2}, \ldots, p_{g D}\right)$ is the most optimal position which has been experienced by the whole particle swarm, then particle [13] updates velocity $v$ and position $z$ according to the following equations during the iterations:

$$
\begin{gathered}
v_{i d}^{k+1}=v_{i d}^{k}+c_{1} r_{1}\left(p_{i d}-z_{i d}^{k}\right)+c_{2} r_{2}\left(p_{g d}-z_{i d}^{k}\right), \\
z_{i d}^{k+1}=z_{i d}^{k}+v_{i d}^{k+1},
\end{gathered}
$$

where $k$ is the iteration times; $r_{1}$ and $r_{2}$ are random numbers in range of $[0,1]$ and they will keep the diversity of the swarm; $c_{1}$ and $c_{2}$ are learning factors, and they make the particle have the ability to summarize itself and learn from the excellent individual in the whole particle swarm, and finally the particle closes to the optimal positioning in its history and in the whole particle swarm history.

To improve the movement velocity of particle and enhance the local searching ability, PSO with convergence factor $\mu$ is commonly used. The velocity $v$ and position $z$ are as follows [14]:

$$
\begin{gathered}
v_{i d}^{k+1}=\mu\left[v_{i d}^{k}+c_{1} r_{1}\left(p_{i d}-z_{i d}^{k}\right)+c_{2} r_{2}\left(p_{g d}-z_{i d}^{k}\right)\right], \\
z_{i d}^{k+1}=z_{i d}^{k}+v_{i d}^{k+1},
\end{gathered}
$$

where

$$
\begin{gathered}
\mu=\frac{2}{\left|2-l-\sqrt{l^{2}-4 l}\right|}, \\
l=c_{1}+c_{2}, \quad l>4,
\end{gathered}
$$

$k$ is the number of iteration.
The convergence factor $\mu$ and its parameter $l$ can control the velocity. The optimal position, $p_{i}=\left(p_{i 1}, p_{i 2}, \ldots, p_{i D}\right)$, is experienced so far by the $i$ th individual particle. The optimal position, $p_{g}=\left(p_{g 1}, p_{g 2}, \ldots, p_{g D}\right)$, is experienced so far by the whole particle swarm $[12,13]$.

To keep the balance between the global searching and the local searching, and decrease the iteration number for optimal solution, the inertia PSO (IPSO) is usually used. The velocity $v$ and position $z$ of IPSO can be described as follows [15]:

$$
\begin{gathered}
v_{i d}^{k+1}=w v_{i d}^{k}+c_{1} r_{1}\left(p_{i d}-z_{i d}^{k}\right)+c_{2} r_{2}\left(p_{g d}-z_{i d}^{k}\right), \\
z_{i d}^{k+1}=z_{i d}^{k}+v_{i d}^{k+1},
\end{gathered}
$$

where $w$ is inertia factor which plays a role of a tradeoff between global optimization and local optimization.

The probability of the global optimization can be increased with a fewer iteration number by applying the IPSO.

\section{NICCM Classifier}

As we know from the above section, the punishment factor $C$ and the kernel parameter $\sigma$ should be optimized to ensure the quality of SVM classification model. That is to say, the parameter selection processes of SVM are the optimal searching processes, and each point in the searching space is a potential solution of the optimal model $[16,17]$.

3.1. Definition of NICPSO. In order to make use of the advantages of the PSO with inertia factor and the PSO with convergence factor, these two PSOs are fused to construct the NICPSO. The NICPSO velocity of a particle is defined as the weighted velocity of the inertia PSO:

$$
v_{i d}^{k+1}=\mu\left[w_{n} v_{i d}^{k}+c_{1} r_{1}\left(p_{i d}-z_{i d}^{k}\right)+c_{2} r_{2}\left(p_{g d}-z_{i d}^{k}\right)\right],
$$

where $\mu$ is the convergence factor, $w_{n}$ is the nonlinear inertia factor, and $k$ is the control factor.

Besides, the nonlinear inertia factor $w_{n}$ is selected to be

$$
w_{n}(t)=w_{\min }+\left(w_{\max }-w_{\min }\right) \exp \left(-m \times\left(\frac{t}{t_{\max }}\right)^{2}\right),
$$

where $t$ is the number of iterations and $m$ is the control factor which control the smoothness degree of the $w$ - $t$ curve. The inertia factor $w_{n}$ varies with the iteration time $t$ in Figure 1. These $w$ - $t$ curves move from the right upper to the left lower when $m=0.5,1.5,5,10,50$. 


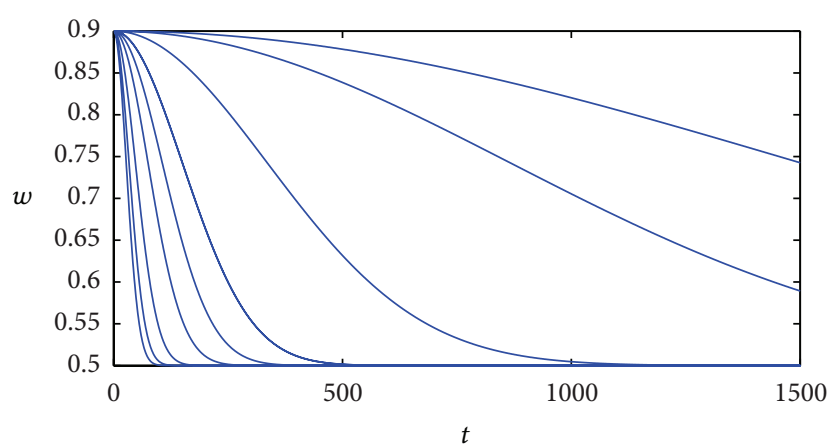

Figure 1: The nonlinear inertia factor $w_{n}$ varies with the number $t$ of iterations for different control factor $m$.

3.2. NICCM Classifier. Based on the above discussion, the NICPSO can be written as

$$
\begin{gathered}
w_{n}(t)=w_{\min }+\left(w_{\max }-w_{\min }\right) \exp \left(-m \times\left(\frac{t}{t_{\max }}\right)^{2}\right), \\
v_{i d}^{k+1}=\mu\left[w_{n} v_{i d}^{k}+c_{1} r_{1}\left(p_{i d}-z_{i d}^{k}\right)+c_{2} r_{2}\left(p_{g d}-z_{i d}^{k}\right)\right], \\
z_{i d}^{k+1}=z_{i d}^{k}+v_{i d}^{k+1},
\end{gathered}
$$

where $w_{n}$ is the nonlinear inertia factor of NICPSO, $\mu$ is the convergence factor, $v$ is the speed of particle, and $z$ is the position of particle.

To optimize the punishment factor $C$ and the kernel parameter $\sigma$, the proposed NICPSO and the NICCM are used. As we know, these two parameters have great influence on the quality of SVM. The NICCM $f(x)$ is given as

$$
\begin{gathered}
f(x)=\operatorname{sgn}\left\{\sum_{i=1}^{N} y_{i} \alpha_{i} K\left(x, x_{i}\right)+b\right\}, \\
0 \leq \alpha_{i} \leq C, \quad i=1, \ldots, N, \\
K\left(x, x_{i}\right)=e^{-\left\|x-x_{i}\right\|^{2} /\left(2 \sigma^{2}\right)},
\end{gathered}
$$

where $x_{i}$ and $y_{i}$ are, respectively, the $i$ th pattern and its class label, $\alpha_{i}$ is the $i$ th Lagrange multiplier, $b$ is the constant of the classifier, and $N$ is the number of samples.

The flow chart of NICCM is given in Figure 2.

The NICCM can be described as follows.

Step 1. Initialize the parameters of PSO.

Step 2. Evaluate every particle.

Step 3. Update the velocity and the position of each particle.

Step 4. Keep the particles as the optimal particles if the searching result is satisfied. Otherwise, go back to Step 2 .

Step 5. Train the NICCM classifier using the optical punishment factor and the optical kernel parameter that comes from the optimal particle.

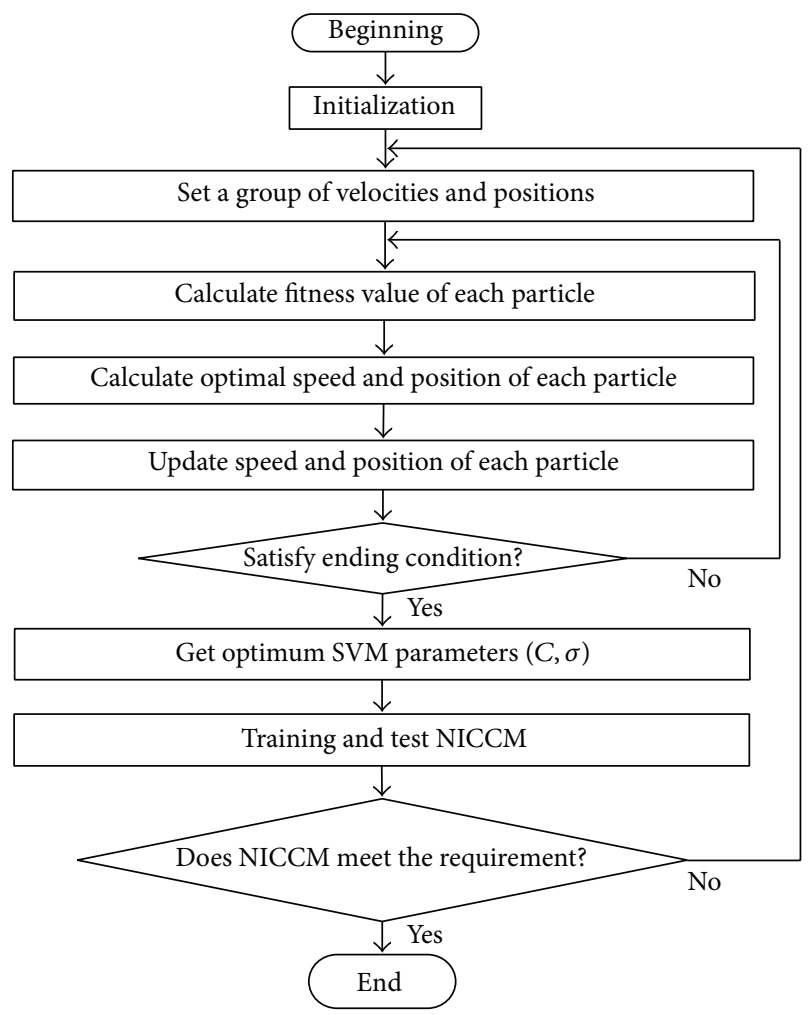

FIgURE 2: Flow chart of NICCM.

Step 6. Keep the trained NICCM as the classification model and end the training process if the performance is satisfied. Otherwise go back to Step 5.

Some parameters are set in Step 1. They are the size $N$ of PSO, the accelerators $c_{1} \& c_{2}$, the maximum velocity $v_{\max }$, and the maximum position $p_{\max }$. The particle is constructed by a punishment factor and a kernel parameter. Namely, the particle is a vector $(C, \sigma)$.

For the particle evaluation, the fitness value of each particle is calculated according to fitness function. The fitness value of each particle is compared with the best one of all its experienced positions. If the comparison result is better, then this position is taken as the best local one instead of the old best local one. Furthermore, if a fitness value is better than the best one among all the particles, then this position is taken as the best global one instead of the old best global one.

\section{Simulated Experiments}

In this section, we recognize the normal state and the fault states of an online power cable by using NICPSO and NICCM. The fault states include the short circuit between phases (SCBP), the three-phase shirt circuit (TPSC), and the normal state (NS).

4.1. Experimental Data. In the system model of the online cable in Figure 3, the two power supplies are ideal, and they provide voltage of $6000 \mathrm{KV}$. The module of three-phase fault 


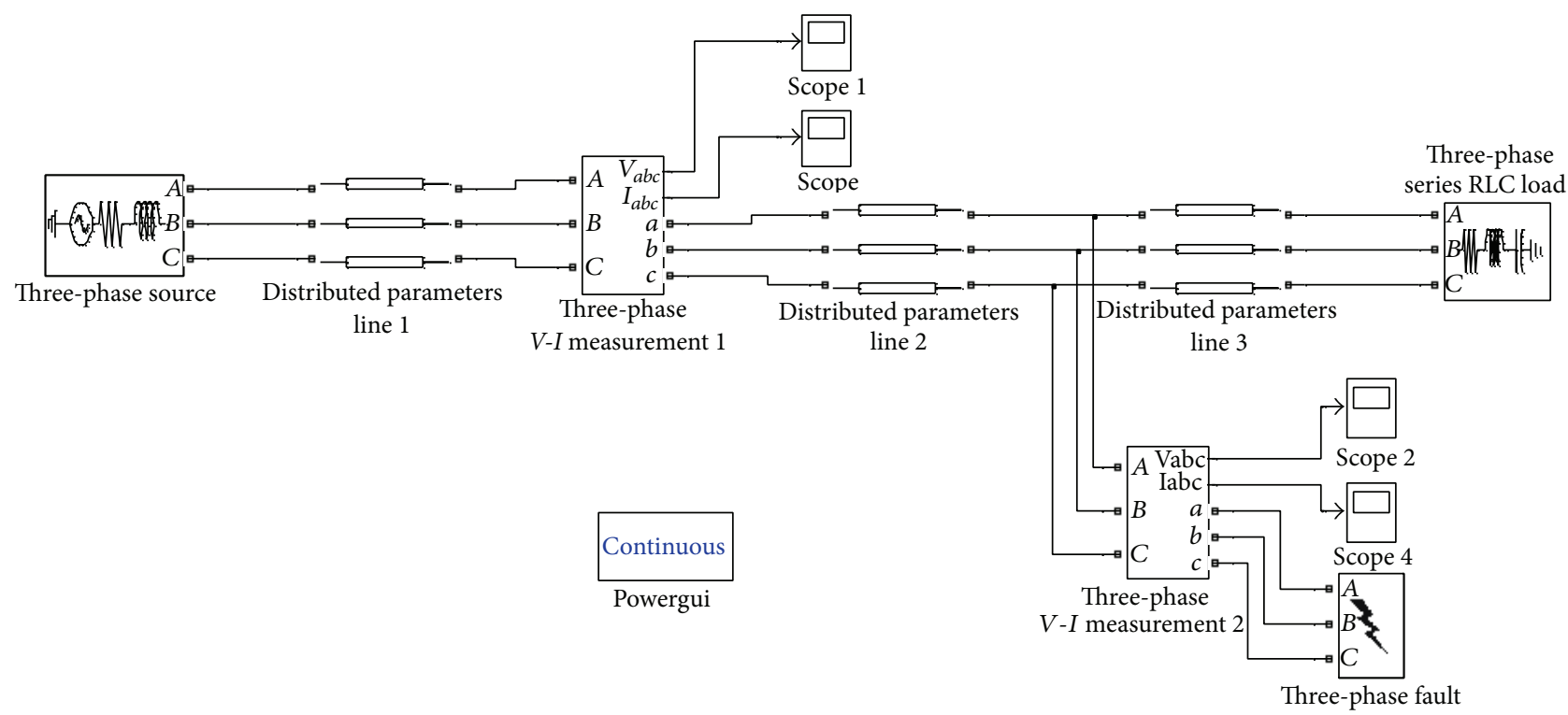

FIGURE 3: System model of online power cable.

is used to produce various types of fault states. The module of three-phase $V-I$ measurement is connected with the power line in front of the fault module to measure the transient fault signals. The sampling frequency is $10 \mathrm{MHz}$. The 1024 samples are collected after the initial traveling waves arrived. The fault starts at the 0.03th second and ends at the 0.07 th second. The total length of the power cable line is $60 \mathrm{~km}$.

The patterns of the states of online power cable system can be constructed by two kinds of features of phase entropy and amplitude entropy. We select the phase entropy $e_{p}$ and the amplitude entropy $e_{a}$ as follows:

$$
\begin{gathered}
e_{p}=\ln \left|-\sum_{k} E_{k} \ln E_{k}\right|, \\
E_{k}=\sum_{j} d_{k}^{2}(p), \\
e_{a}=\ln \left|-\sum_{k} E_{k} \ln E_{k}\right|, \\
E_{k}=\sum_{j} d_{k}^{2}(a),
\end{gathered}
$$

where $E_{k}$ is the energy function of the $k$ th frequency band at scale $j$ of the zero-component of 3-phase currents and $d_{k}$ is the coefficient of the $k$ th frequency band of wavelet packet decomposition [16].

The experimental sample numbers are given in Table 1 and partial sample data are shown in Table 2. The classes of the data include the SCBP, the TPSC, and the NS. The first dimension represents the phase entropy $e_{p}$, and the second dimension represents the amplitude entropy $e_{a}$ in the 2 dimensional vectors of Table 2.

The distribution of the sample data is shown in Figure 4. The red symbols " $x$ " represent the training data of SCBP and

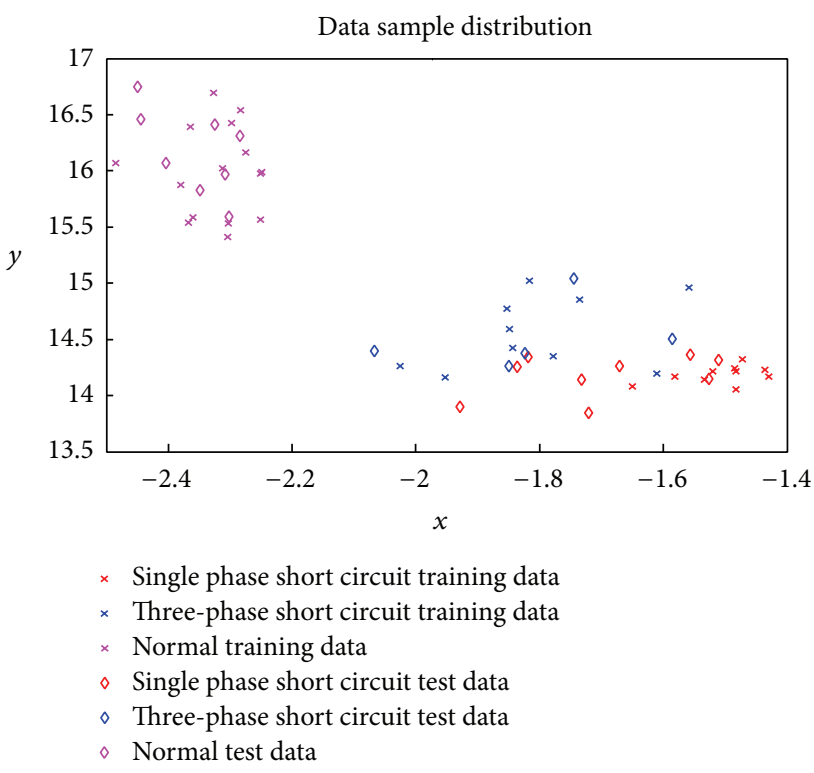

FIGURE 4: Distribution of the sample data.

TABLE 1: Sample number.

\begin{tabular}{lccc}
\hline Class & $\begin{array}{c}\text { Number of } \\
\text { training samples }\end{array}$ & $\begin{array}{c}\text { Number of test } \\
\text { samples }\end{array}$ & $\begin{array}{c}\text { Total number of } \\
\text { samples }\end{array}$ \\
\hline SCBP & 11 & 9 & 20 \\
TPSC & 10 & 5 & 15 \\
NS & 15 & 10 & 25 \\
Sum & 36 & 24 & 60 \\
\hline
\end{tabular}

the blue symbols " $x$ " are the training data of TPSC and the purple symbols " $x$ " are the training data of NS. Meanwhile, the red symbols " $\diamond$ " are the test data of SCBP, the blue 


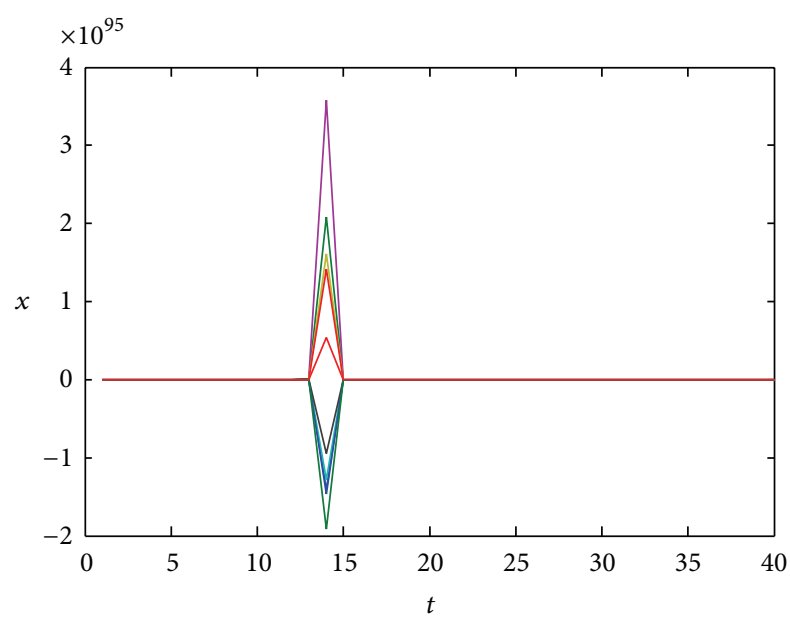

FIgURE 5: Position change of $\mu=1, w=1$.

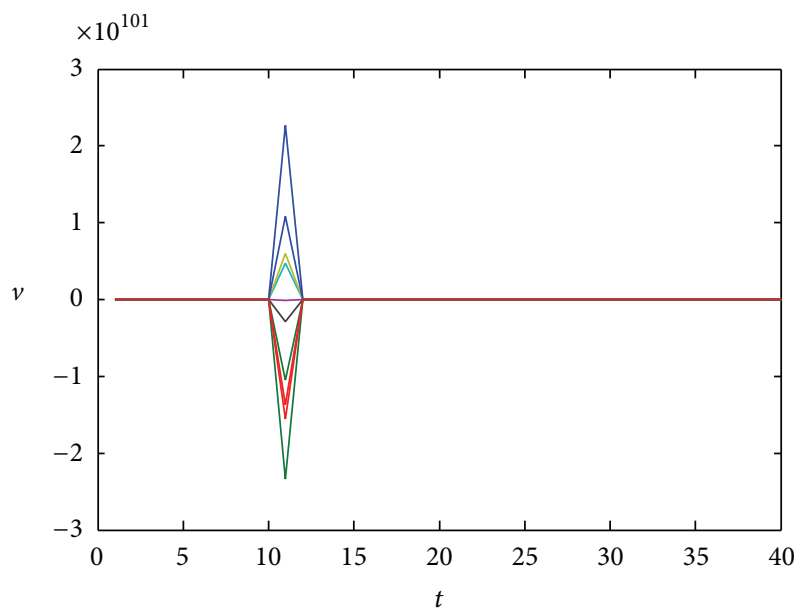

FIGURE 6: Velocity change of $\mu=1, w=1$.

symbols " $\diamond$ " are the test data of TPSC, and purple symbols " $\diamond$ " are the test data of NS.

4.2. NICCM Experiment. The position changes and velocity changes of NICPSO are shown in Figures 5, 6, 7, 8, 9, 10, and 11 with different convergence factor $\mu$ and different inertia factor $w$.

The changes of position and velocity are, respectively, in Figures 5 and 6 for $\mu=1, w=1$. Furthermore, the changes are in Figures 7 and 8 for $\mu=1, w \neq 1$. Figures 9 and 10 are for $\mu \neq 1, w=1$. Figures 11 and 12 are for $\mu \neq 1, w=1$.

From Figures 5 and 11, it can be seen that the iteration numbers of position decrease from 15 times to 5 times for NICPSO to compare with SVM.

From Figures 6 and 12, it can be seen that the iteration numbers of velocity are the same for SVM and NICPSO. The value of both SVM and NICPSO is 12 .

The classification model of SVM is trained and shown in Figure 13. Obviously, all the training samples of NS, SCBP, and TPSC are classified perfectly.

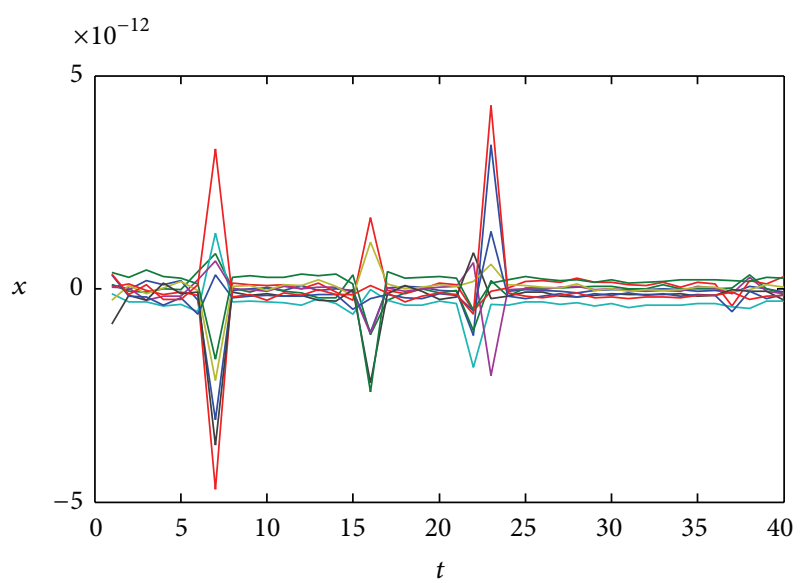

FIgURE 7: Position change of $\mu=1, w \neq 1$.

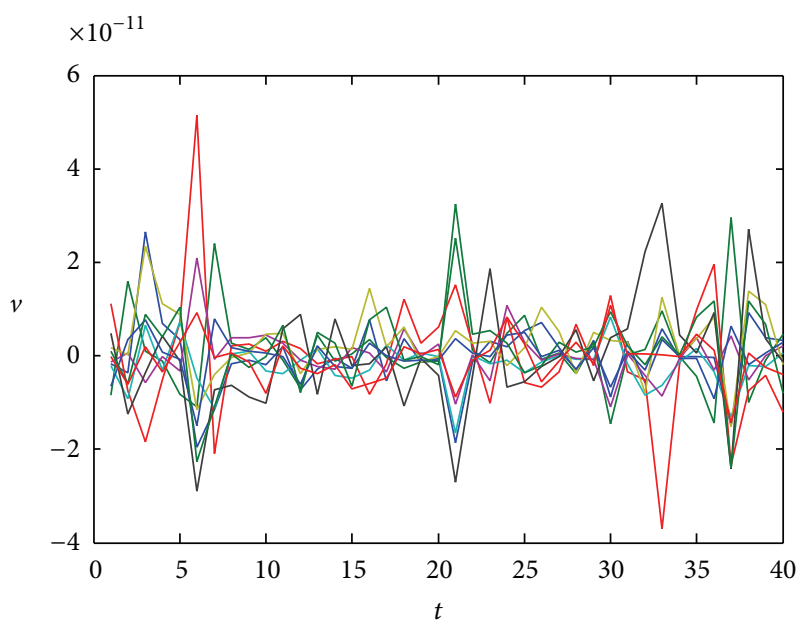

FIgURE 8: Velocity change of $\mu=1, w \neq 1$.

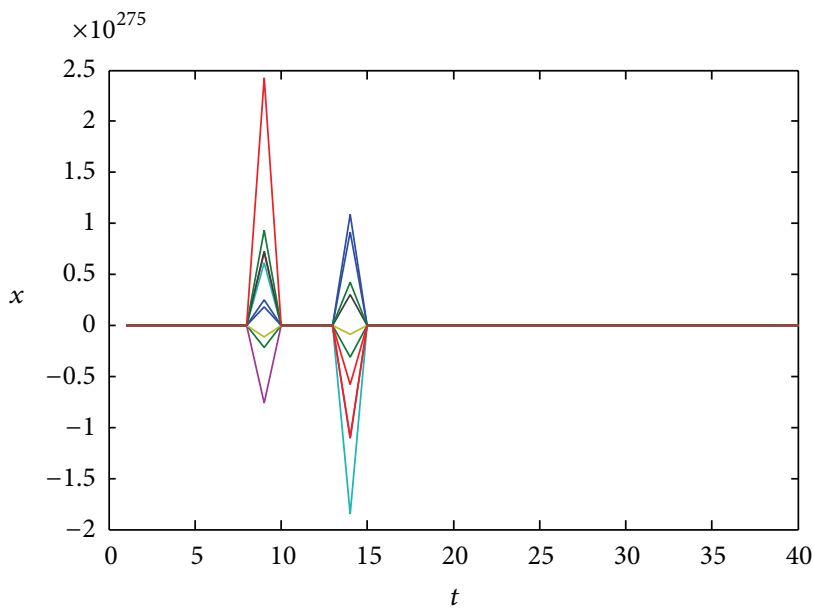

FIgURE 9: Position change of $\mu \neq 1, w=1$. 


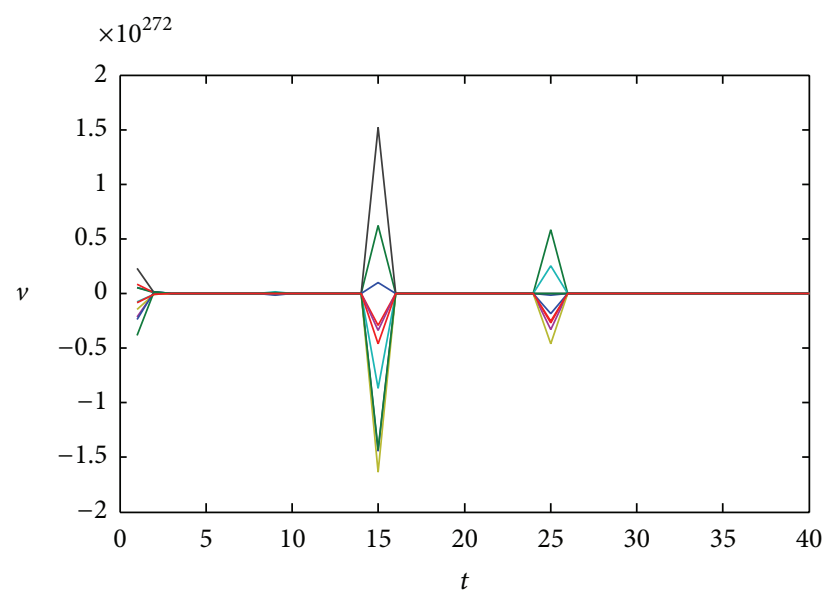

Figure 10: Velocity change of $\mu \neq 1, w=1$.

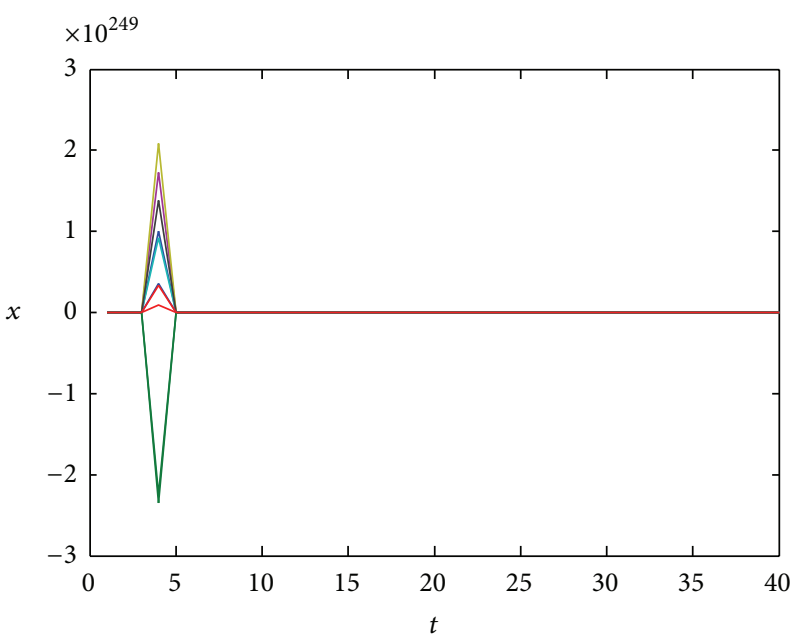

FIGURE 11: Position change of $\mu \neq 1, w \neq 1$.

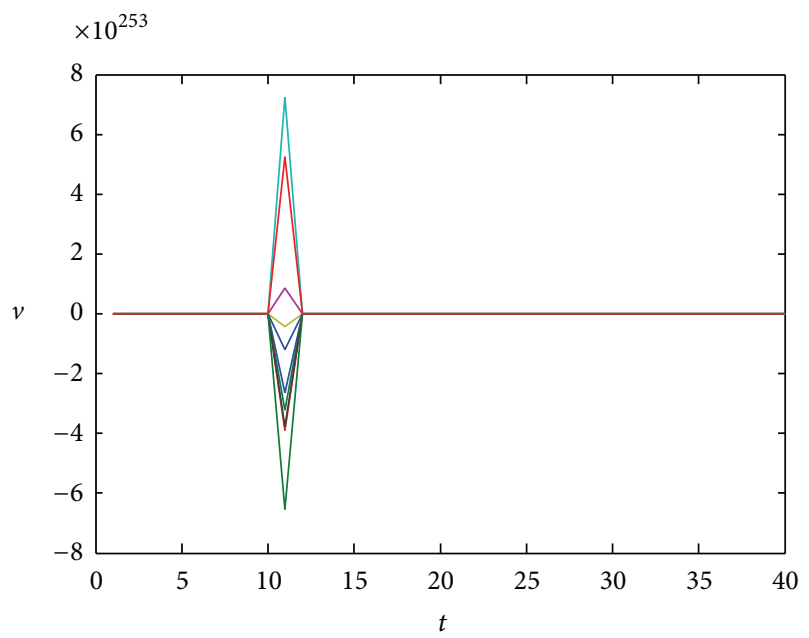

FIGURE 12: Velocity change of $\mu \neq 1, w \neq 1$.

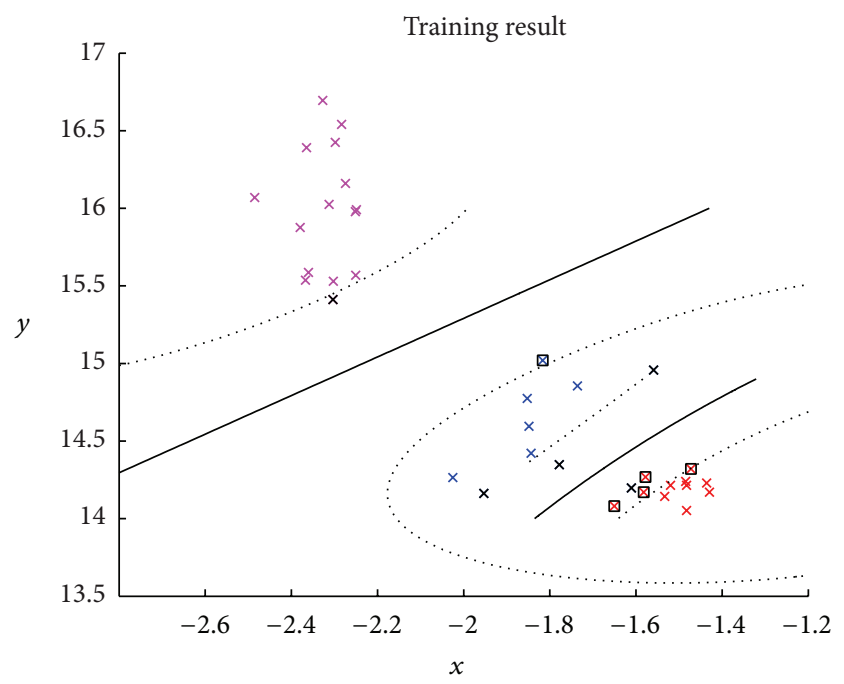

FIGURE 13: Classification model of NS, SCBP, and TPSC using SVM.

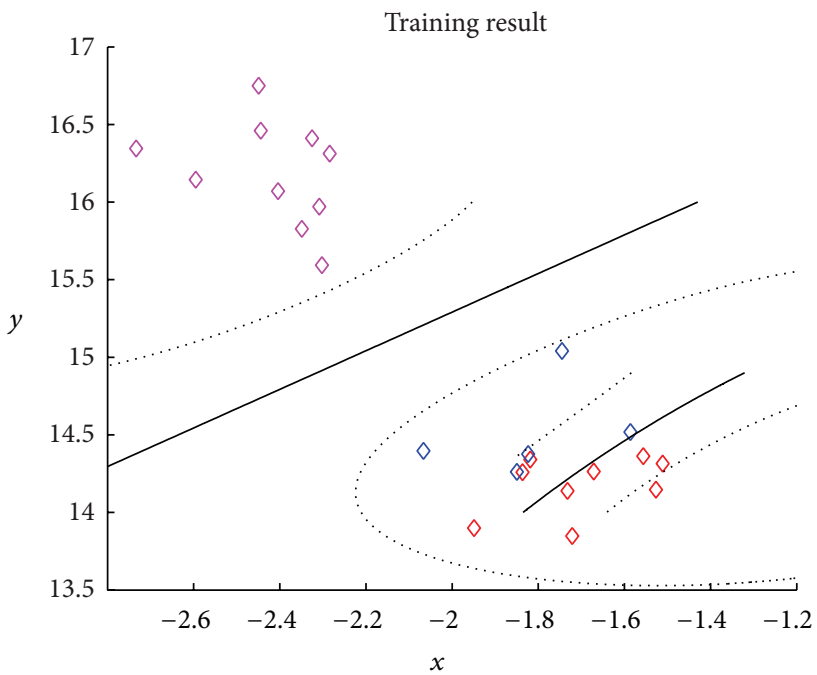

FIGURE 14: Test result of NS, SCBP, and TPSC using SVM.

The test result of SVM model is shown in Figure 14. Training duration is $0.0575 \mathrm{~s}$, and the recognition precision is $87.55 \%$.

The classification model of NICCM is trained and shown in Figure 15. Obviously, all the training samples of NS, SCBP, and TPSC are classified correctly.

The test result of NICCM model is shown in Figure 16. Training duration is $0.0523 \mathrm{~s}$, and it decreases by $0.0052 \mathrm{~s}$ compared with SVM. Furthermore, the test results illustrate that all the samples of NS are classified correctly, and all the faulty samples of SCBP and TPSC are confirmed as faulty state. The shortcoming is that 2 samples of SCBT are recognized as TPSC, and 1 sample cannot be confirmed whether it belongs to SCBT or TPSC. The recognition precision is $91.67 \%$, and it increases by $4.12 \%$ compared with SVM.

4.3. Evaluation. The results obtained from the above experiments are shown in Tables 3 and 4. 
TABLE 2: Partial sample data.

\begin{tabular}{lcc}
\hline Class & Data of training samples & Data of test samples \\
\hline & $(-1.4826,14.2146)$ & $(-1.7212,13.8477)$ \\
& $(-1.4725,14.3209)$ & $(-1.5111,14.3149)$ \\
& $(-1.5333,14.1438)$ & $(-1.5563,14.3628)$ \\
& $(-1.4359,14.2299)$ & $(-1.5268,14.1465)$ \\
SCBP & $(-1.5196,14.2148)$ & $(-1.7323,14.1386)$ \\
& $(-1.4826,14.0529)$ & $(-1.6713,14.2635)$ \\
& $(-1.4848,14.2394)$ & $(-1.9292,13.8993)$ \\
& $(-1.4295,14.1701)$ & $(-1.8366,14.2588)$ \\
& $(-1.5818,14.1710)$ & $(-1.8185,14.3409)$ \\
\hline & $(-1.8482,14.5948)$ & $(-2.0666,14.3968)$ \\
TPSC & $(-1.7781,14.3483)$ & $(-1.7447,14.0400)$ \\
& $(-1.8164,14.0203)$ & $(-1.8236,14.3761)$ \\
& $(-2.0253,14.2646)$ & $(-1.8498,14.2612)$ \\
& $(-1.8436,14.4213)$ & $(-1.5861,14.5057)$ \\
\hline & $(-2.4852,16.0696)$ & $(-2.3082,15.9704)$ \\
& $(-2.3795,15.8763)$ & $(-2.2841,16.3121)$ \\
& $(-2.3672,15.5368)$ & $(-2.4491,16.7497)$ \\
NS & $(-2.3647,16.3910)$ & $(-2.3021,15.5935)$ \\
& $(-2.2980,16.4249)$ & $(-2.5951,16.1439)$ \\
& $(-2.3126,16.0252)$ & $(-2.3489,15.8276)$ \\
& $(-2.3036,15.4120)$ & $(-2.7336,16.3454)$ \\
& $(-2.2745,16.1606)$ & $(-2.3253,16.4116)$ \\
$(-2.2515,15.9785)$ & $(-2.4441,16.4604)$ \\
& $(-2.2507,15.5681)$ & $(-2.4040,16.0707)$ \\
\hline & &
\end{tabular}

TABle 3: Comparison of different PSOs.

\begin{tabular}{lccc}
\hline Method & $\begin{array}{c}\text { Parameter } \\
\text { and factor }\end{array}$ & $\begin{array}{c}\text { Iteration number } \\
\text { of velocity }\end{array}$ & $\begin{array}{c}\text { Iteration number } \\
\text { of position }\end{array}$ \\
\hline PSO & $u=1, w=1$ & 12 & 15 \\
IPSO & $u=1, w \neq 1$ & $>40$ & 39 \\
CPSO & $u \neq 1, w=1$ & 26 & 15 \\
NICPSO & $u \neq 1, w \neq 1$ & 12 & 5 \\
\hline
\end{tabular}

TABLE 4: Evaluation 1 of NICCM.

\begin{tabular}{lcc}
\hline Method & Training duration & Recognition precision \\
\hline SVM & 0.0575 & $87.55 \%$ \\
NICCM & 0.0523 & $91.67 \%$ \\
\hline
\end{tabular}

\section{Conclusion}

In this paper, the NICPSO is developed to optimize the punishment factor and the kernel parameter of SVM, and then the NICCM is proposed to implement classification. Finally, the NICCM is applied to classify the normal state and fault states of online power cable. It is experimentally proved that the iteration number for the proposed NICPSO to reach the optimal position decreases from 15 to 5 compared with the conventional PSO. All the samples of normal state are classified correctly, and all the samples of fault state are confirmed by using NICCM. Compared with SVM,

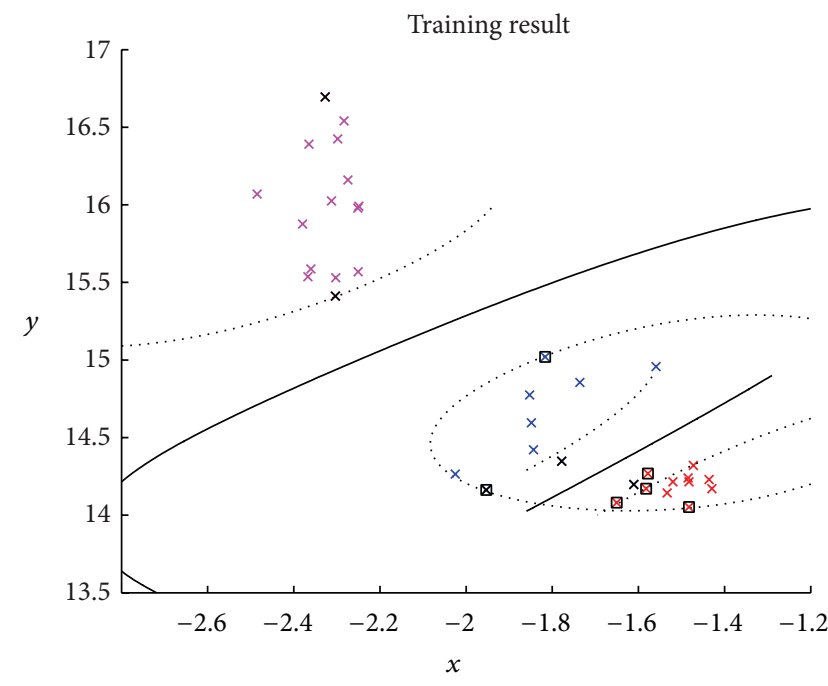

FIGURE 15: Classification model of NS, SCBP, and TPSC using NICCM.

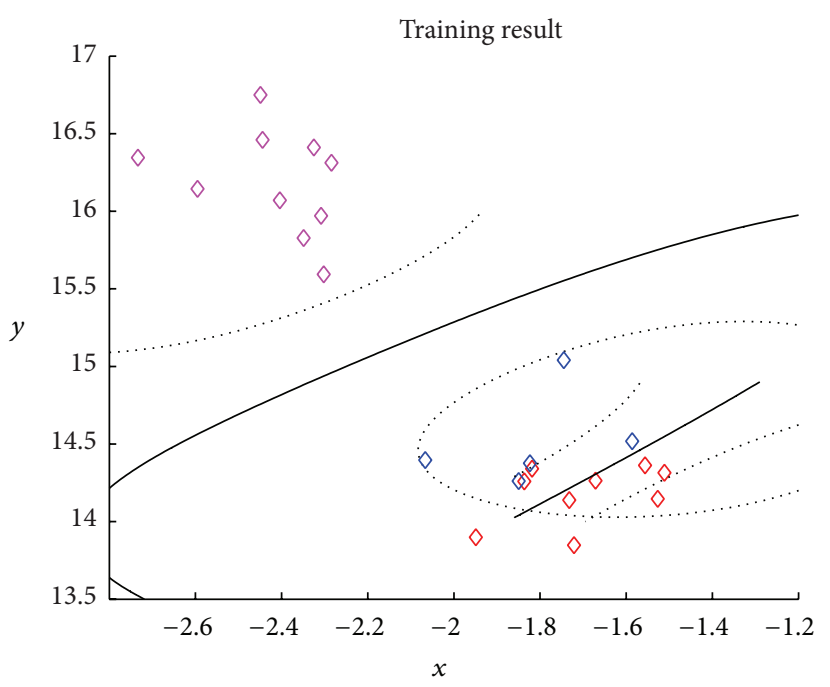

FIGURE 16: Test result of NS, SCBP, and TPSC using NICCM.

the training duration of NICCM is decreased by $0.0052 \mathrm{~s}$. Meanwhile, the recognition precision of NICCM is increased by $4.12 \%$.

NICCM is not only suitable for the classification of the normal state and fault states of online power cable but also suitable for other classifications with a small number of samples and a quick recognition process.

\section{Conflict of Interests}

The authors declare that there is no conflict of interests regarding the publication of this paper. 


\section{Acknowledgments}

This research is sponsored by Scientific Research Foundation for Returned Scholars, Ministry of Education of China ([2011]508), and Natural Science Foundation of Shaanxi Province (2011JM8005).

\section{References}

[1] A. J. Reid, C. Zhou, D. M. Hepburn, M. D. Judd, W. H. Siew, and P. Withers, "Fault location and diagnosis in a medium voltage EPR power cable," IEEE Transactions on Dielectrics and Electrical Insulation, vol. 20, no. 1, pp. 10-18, 2013.

[2] M. Wang and T. Stathaki, "Online fault recognition of electric power cable in coal mine based on the minimum risk neural network," Journal of Coal Science \& Engineering, vol. 14, no. 3, pp. 492-496, 2008.

[3] M. Z. Salvador, R. G. Resmini, and R. B. Gomez, "Detection of sulfur dioxide in AIRS data with the wavelet packet subspace," IEEE Geoscience and Remote Sensing Letters, vol. 6, no. 1, pp. 137-141, 2009.

[4] S. M. J. Rastegar Fatemi, J. Soltani, N. R. Abjadi, and G. R. A. Markadeh, "Space-vector pulse-width modulation of a Zsource six-phase inverter with neural network classification," IET Power Electronics, vol. 5, no. 9, pp. 1956-1967, 2012.

[5] G. T. Kaya, "A hybrid model for classification of remote sensing images with linear SVM and support vector selection and adaptation," IEEE Journal of Applied Earth Observations and Remote Sensing, vol. 6, no. 4, pp. 1988-1997, 2013.

[6] Y.-L. Li, W. Shao, L. You, and B.-Z. Wang, "An improved PSO algorithm and its application to UWB antenna design," IEEE Antennas and Wireless Propagation Letters, vol. 12, pp. 12361239, 2013.

[7] R. Baly and H. Hajj, "Wafer classification using support vector machines," IEEE Transactions on Semiconductor Manufacturing, vol. 25, no. 3, pp. 373-383, 2012.

[8] Y. Sela, M. Freiman, E. Dery et al., "FMRI-based hierarchical SVM model for the classification and grading of liver fibrosis," IEEE Transactions on Biomedical Engineering, vol. 58, no. 9, pp. 2574-2581, 2011.

[9] M. Wang, Z. Dan, and L. Wang, "Power cable faults diagnosis based on the convex hull binary tree SVM," in Proceedings of the 2nd International Congress on Image and Signal Processing (CISP '09), pp. 1-5, Tianjin, China, October 2009.

[10] J. Xiao and J. Lin, "Support vector classification algorithm based on variable parameter linear programming," Journal of Systems Engineering and Electronics, vol. 18, no. 2, pp. 355-359, 2007.

[11] Z. Li, J. Zhang, and S. Hu, "Incremental support vector machine algorithm based on multi-kernel learning," Journal of Systems Engineering and Electronics, vol. 22, no. 4, pp. 702-706, 2011.

[12] A. Modiri and K. Kiasaleh, "Modification of real-number and binary PSO algorithms for accelerated convergence," IEEE Transactions on Antennas and Propagation, vol. 59, no. 1, pp. 214-224, 2011.

[13] R. A. Hooshmand and S. Soltani, "Fuzzy optimal phase balancing of radial and meshed distribution networks using BF-PSO algorithm," IEEE Transactions on Power Systems, vol. 27, no. 1, pp. 47-57, 2012.

[14] W. Ding, J. Wang, and Z. Guan, "Cooperative extended rough attribute reduction algorithm based on improved PSO," Journal of Systems Engineering and Electronics, vol. 23, no. 1, pp. 160-166, 2012.
[15] J. B. Park, Y. W. Jeong, J. R. Shin, and K. Y. Lee, “An improved particle swarm optimization for nonconvex economic dispatch problems," IEEE Transactions on Power Systems, vol. 25, no. 1, pp. 156-166, 2010.

[16] É. J. de Oliveira, I. M. da Fonseca, and H. K. Kuga, "Fault detection and isolation in inertial measurement units based on $\chi^{2}$-CUSUM and wavelet packet," Mathematical Problems in Engineering, vol. 2013, Article ID 869293, 10 pages, 2013.

[17] W. Hu, J. Gao, Y. Wang, O. Wu, and S. Maybank, "Online adaboost-based parameterized methods for dynamic distributed network intrusion detection," IEEE Transactions on Cybernetics, vol. 44, no. 1, pp. 66-82, 2014. 


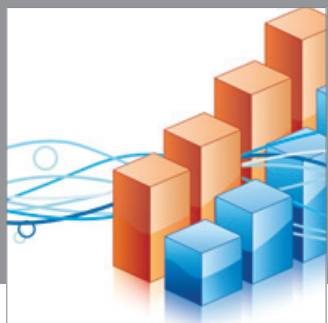

Advances in

Operations Research

mansans

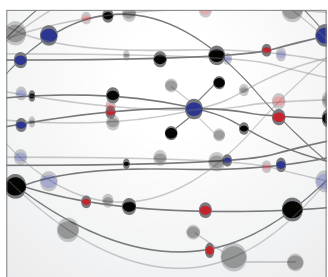

The Scientific World Journal
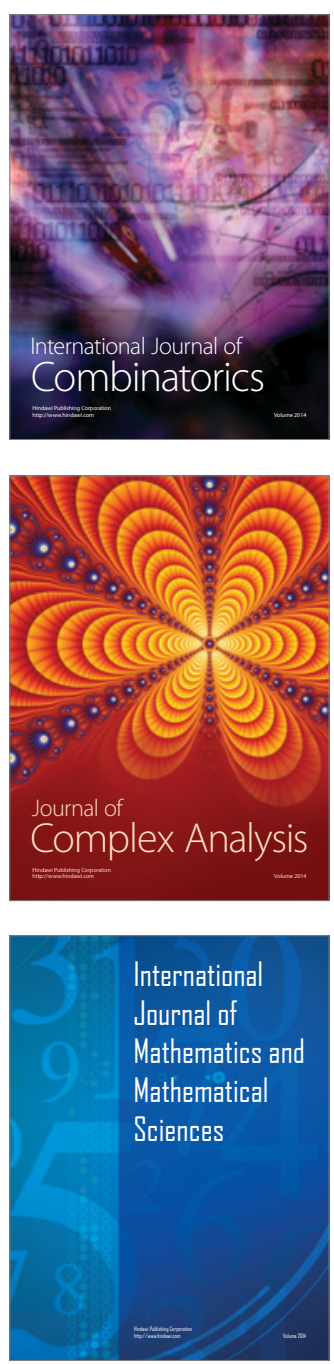
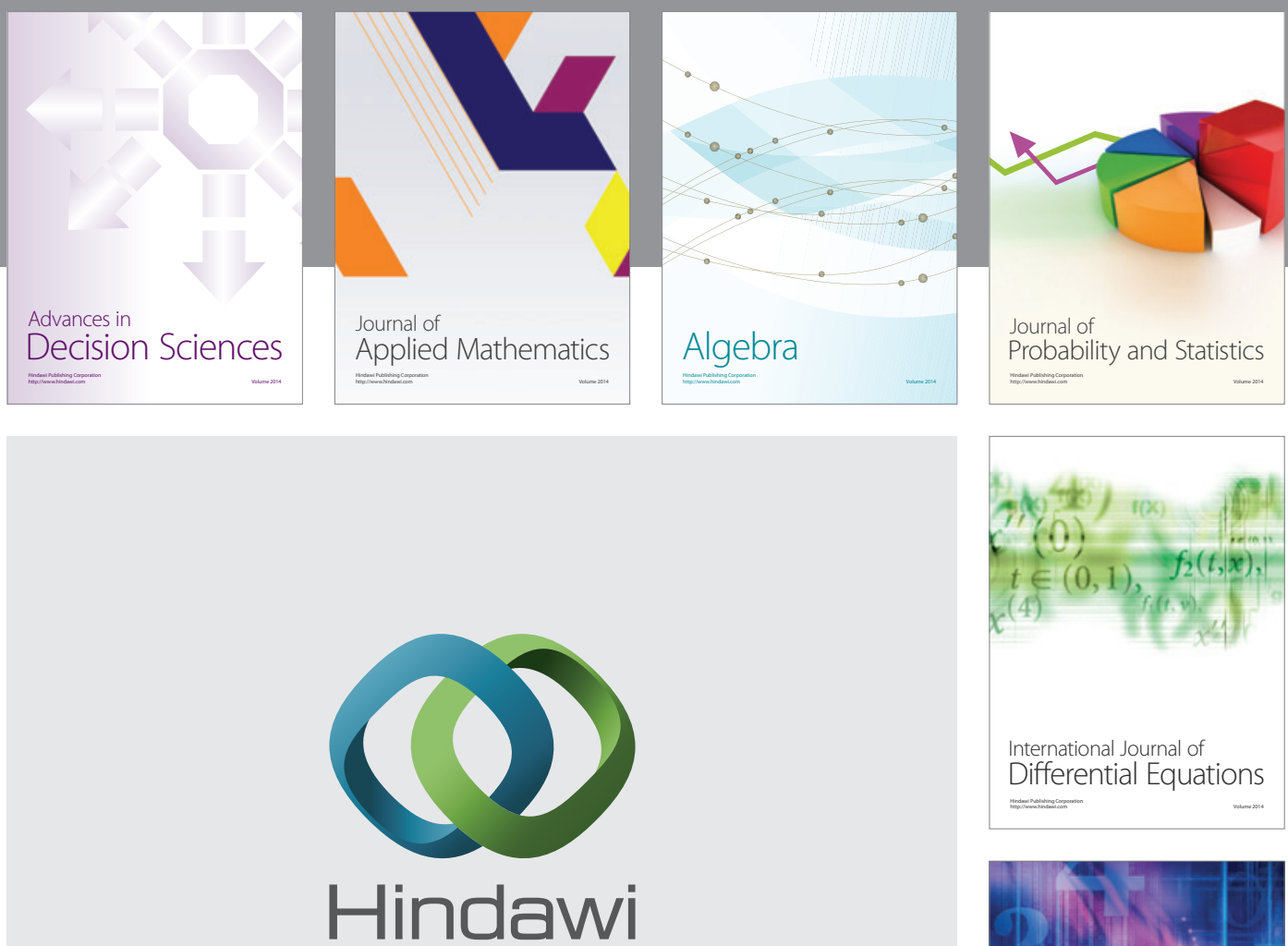

Submit your manuscripts at http://www.hindawi.com
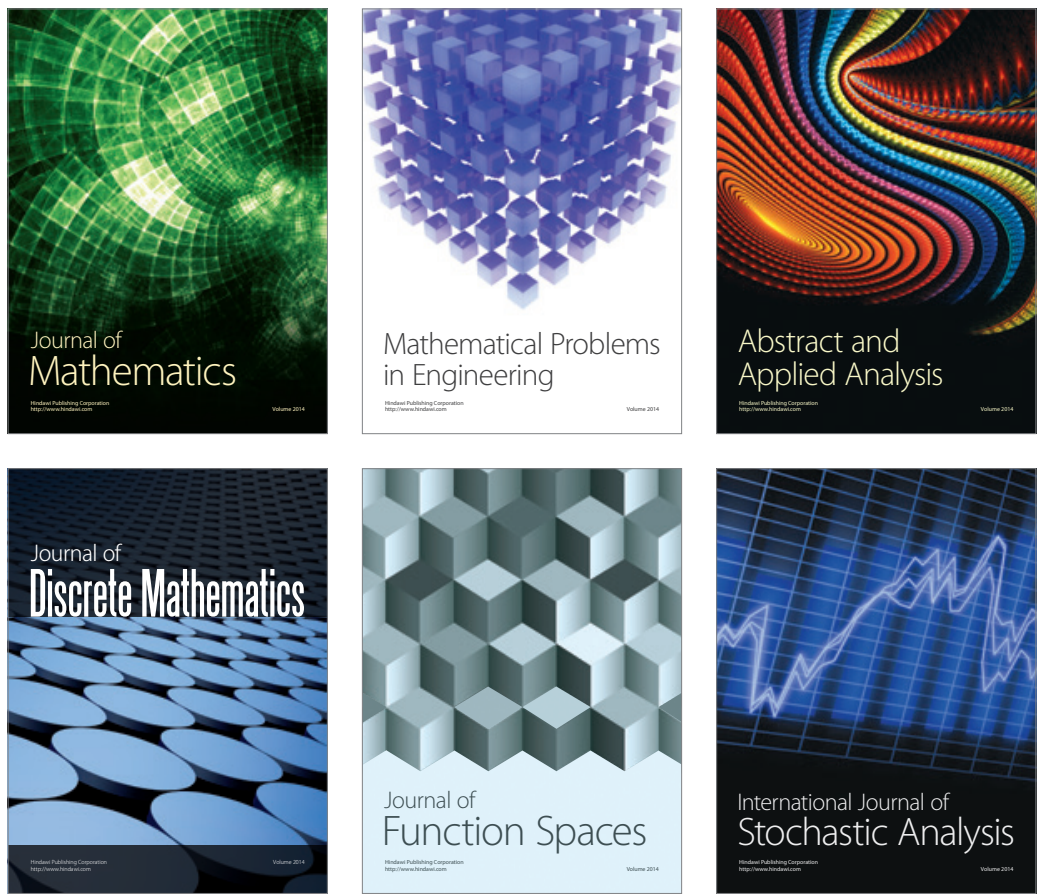

Journal of

Function Spaces

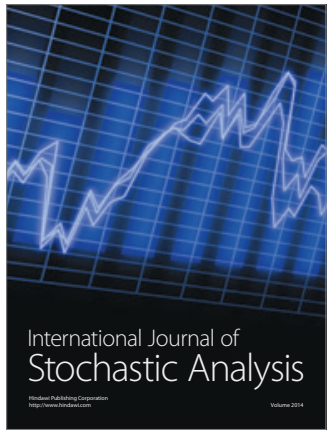

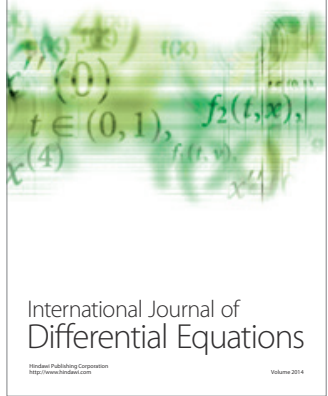
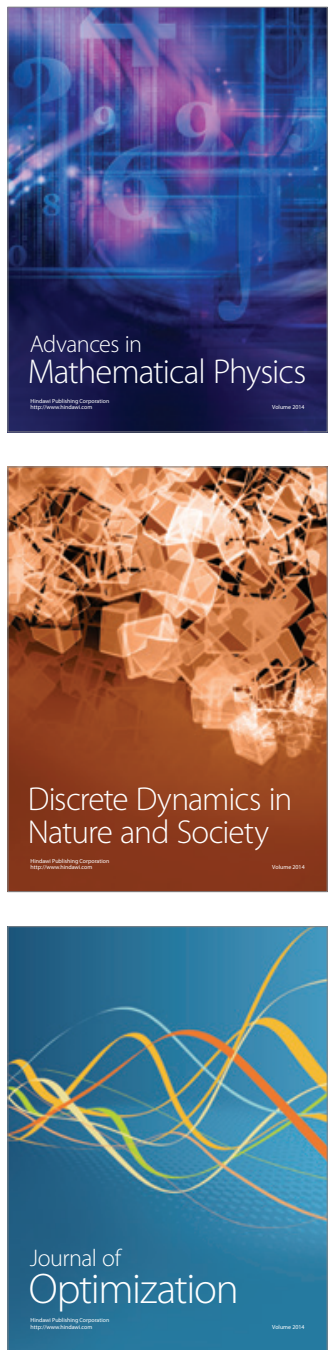